\title{
On unique solvability of quadratic integral equations with linear modification of the argument
}

Mouffak Benchohra and Mohamed Abdalla Darwish 


\title{
ON UNIQUE SOLVABILITY OF QUADRATIC INTEGRAL EQUATIONS WITH LINEAR MODIFICATION OF THE ARGUMENT
}

\author{
MOUFFAK BENCHOHRA AND MOHAMED ABDALLA DARWISH
}

Received 3 September, 2007

\begin{abstract}
In this paper, we investigate the existence of a unique solution on a semi-infinite interval for quadratic integral equations with linear modification of the argument in Fréchet spaces using a nonlinear alternative of Leray-Schauder type for contraction maps in Fréchet spaces.
\end{abstract}

2000 Mathematics Subject Classification: 45G10, 45M99, 47H09, 47H10

Keywords: quadratic integral equations, linear modification of the argument, existence and uniqueness, fixed-point, contraction maps, nonlinear alternative of Leray-Schauder type, Fréchet space

\section{INTRODUCTION}

In this paper, we establish the existence of the unique solution, defined on a semiinfinite interval $J:=[0,+\infty)$ for the following quadratic integral equations with a linear modification of the argument

$$
x(t)=f(t)+(A x)(t) \int_{0}^{T} u(t, s, x(s), x(\alpha s)) d s, \quad t \in J,
$$

and

$$
x(t)=f(t)+g(t, x(t)) \int_{0}^{T} u(t, s, x(s), x(\alpha s)) d s, \quad t \in J,
$$

where $f: J \rightarrow \mathbb{R}, g: J \times \mathbb{R} \rightarrow \mathbb{R}, u: J \times J_{T} \times \mathbb{R}^{2} \rightarrow \mathbb{R}$ are given functions, $0<\alpha<1$, $J_{T}:=[0, T]$, and $A: C(J ; \mathbb{R}) \rightarrow C(J ; \mathbb{R})$ is an appropriate operator. Here $C(J ; \mathbb{R})$ denotes the space of continuous functions $x: J \rightarrow \mathbb{R}$.

Integral equations arise naturally in many applications in describing numerous real world problems (see, for instance, the books $[1,2,8,10,20]$ and references therein). Also quadratic integral equations have many useful applications in describing numerous events and problems of the real world. For example, quadratic integral equations are often applicable in the theory of radiative transfer, kinetic theory of gases, in the theory of neutron transport and in the traffic theory. Especially, the so-called quadratic integral equation of Chandrasekher type can be very often encountered in 
many applications; see for instance the book by Chandrasekher [7] and the research papers by Banas et al [3], Benchohra and Darwish [4], Darwish [9], Hu et al [14], Kelley [15], Leggett [17] and Stuart [21] and references therein.

The study of differential equations with a modified argument is relatively new, it was initiated only in the past thirty years or so. These equations arise in the modelling of problems from the natural and social sciences such as biology, economics and physics. A special class is represented by the differential equations with affine modification of the argument which can be differential equations with linear modification of the argument or differential equation with delay. For more information and results concerning these equations, see $[6,11,13,16,18,19]$.

More recently, Caballero et al [5] investigated the so-called quadratic integral equation of the Volterra type with linear modification of the argument, Volterra counter part of equation (1.1), and proved the existence of monotonic solutions in $C([0,1], \mathbb{R})$. There equation can be considered with connection to the following Cauchy problem [5, 19]:

$$
\begin{aligned}
x^{\prime}(t) & =u(t, s, x(t), x(\lambda t)), \quad t \in[0,1], 0<\lambda<1, \\
x(0) & =u_{0} .
\end{aligned}
$$

In this paper, we investigate the question of unique solvability of equation (1.1) and (1.2). Motivated by the previous papers considered for integral equations on a bounded interval, we extend here these results to semi-infinite intervals for a class of quadratic integral equations. The method we are going to use is to reduce the existence of the unique solution for the quadratic integral equation (1.1) to the search for the existence of the unique fixed-point of an appropriate operator on the Fréchet space $C(J ; \mathbb{R})$ by applying a nonlinear alternative of Leray-Schauder type for contraction maps due to Frigon and Granas [12].

\section{Preliminaries}

We introduce notations, definitions, and theorems which are used throughout this paper.

Let $X$ be a Fréchet space with a family of semi-norms $\left\{\|\cdot\|_{n}\right\}_{n \in \mathbb{N}}$. Let $Y \subset X$, we say that $Y$ is bounded if for every $n \in \mathbb{N}$, there exists $M_{n}>0$ such that

$$
\|y\|_{n} \leq M_{n} \quad \text { for every } y \in Y .
$$

To $X$ we associate a sequence of Banach spaces $\left\{\left(X^{n},\|\cdot\|_{n}\right)\right\}$ as follows: For every $n \in \mathbb{N}$, we consider the equivalence relation $\sim_{n}$ defined by the formula

$$
x \sim_{n} y \quad \text { if and only if } \quad\|x-y\|_{n}=0 \quad \text { for all } x, y \in X .
$$

We denote $X^{n}=\left(\left.X\right|_{\sim_{n}},\|\cdot\|_{n}\right)$ the quotient space, the completion of $X^{n}$ with respect to $\|\cdot\|_{n}$. To every $Y \subset X$, we associate a sequence $\left\{Y^{n}\right\}$ of subsets $Y^{n} \subset X^{n}$ as follows: For every $x \in X$, we denote $[x]_{n}$ the equivalence class of $x$ of subset $X^{n}$ and we defined $Y^{n}=\left\{[x]_{n}: x \in Y\right\}$. We denote $\overline{Y^{n}}, \operatorname{Int}_{n}\left(Y^{n}\right)$, and $\partial_{n} Y^{n}$, respectively, 
the closure, the interior and the boundary of $Y^{n}$ with respect to $\|\cdot\|$ in $X^{n}$. We assume that the family of semi-norms $\left\{\|\cdot\|_{n}\right\}$ verifies

$$
\|x\|_{1} \leq\|x\|_{2} \leq\|x\|_{3} \leq \cdots \quad \text { for every } x \in X .
$$

Definition 1 ([12]). A function $f: X \rightarrow X$ is said to be a contraction if for each $n \in \mathbb{N}$ there exists $k_{n} \in(0,1)$ such that

$$
\|f(x)-f(y)\|_{n} \leq k_{n}\|x-y\|_{n} \quad \text { for all } x, y \in X .
$$

Theorem 2.1 ([12]). Suppose that $U$ is an open subset of a Fréchet space $X$, $0 \in U$, and $F: \bar{U} \rightarrow X$ is a contraction such that $F(\bar{U})$ is bounded. Then either,

(C1) $F$ has a unique fixed point in $\bar{U}$; or

(C2) there exist $\lambda \in(0,1)$ and $u \in \partial U$ with the property $u=\lambda F(u)$.

\section{MAIN THEOREM}

In this section, we will study equation (1.1) assuming that the following assumptions are satisfied:

$\left(a_{1}\right) f: J \rightarrow \mathbb{R}$ is a continuous function.

$\left(a_{2}\right)$ For each $n \in \mathbb{N}$ there exists $L_{n}>0$ such that

$|(A x)(t)-(A \bar{x})(t)| \leq L_{n}|x(t)-\bar{x}(t)| \quad$ for each $x, \bar{x} \in C(J ; \mathbb{R})$ and $t \in[0, n]$.

$\left(a_{3}\right)$ There exist nonnegative constants $a$ and $b$ such that

$$
|(A x)(t)| \leq a+b|x(t)|
$$

holds for each $x \in C(J ; \mathbb{R})$ and $t \in J$.

(a $\left.a_{4}\right) u: J \times J_{T} \times \mathbb{R}^{2} \rightarrow \mathbb{R}$ is a continuous function and, for each $n \in \mathbb{N}$, there exists a constant $L_{n}^{*}>0$ such that

$$
|u(t, s, x, y)-u(t, s, \bar{x}, \bar{y})| \leq L_{n}^{*}(|x-\bar{x}|+|y-\bar{y}|)
$$

holds for all $t \in[0, n], s \in J_{T}$, and $x, y, \bar{x}, \bar{y} \in \mathbb{R}$.

$\left(a_{5}\right)$ There exists a continuous nondecreasing function $\psi: J^{2} \rightarrow(0, \infty)$ and $p \in$ $C\left(J ; \mathbb{R}_{+}\right)$such that

$$
|u(t, s, x, y)| \leq p(s) \psi(|x|,|y|)
$$

for each $(t, s) \in J \times J_{T}$ and $x, y \in \mathbb{R}$, and, moreover, there exist constants $M_{n} \in J, n \in \mathbb{N}$, such that

$$
\frac{M_{n}}{\|f\|_{n}+T\left(a+b M_{n}\right) \psi\left(M_{n}, M_{n}\right) p^{*}}>1
$$

holds for all $n \in \mathbb{N}$, where $p^{*}=\sup \left\{p(s): s \in J_{T}\right\}$. 
Theorem 3.1. Let the assumptions $\left(a_{1}\right)-\left(a_{5}\right)$ be satisfied. If, in addition, the inequality

$$
2\left(a+b M_{n}\right) L_{n}^{*} T+T L_{n} \psi\left(M_{n}, M_{n}\right) p^{*}<1
$$

holds for all $n \in \mathbb{N}$, then the equation (1.1) has a unique solution.

Proof. For every $n \in \mathbb{N}$, we define in $C(J ; \mathbb{R})$ the semi-norms by the formula

$$
\|y\|_{n}:=\sup \{|y(t)|: t \in[0, n]\} .
$$

Then $C(J ; \mathbb{R})$ is a Fréchet space with the family of semi-norms $\left\{\|\cdot\|_{n}\right\}_{n \in \mathbb{N}}$.

Transform the problem (1.1) into a fixed-point problem. Consider the operator $\mathcal{F}: C(J ; \mathbb{R}) \rightarrow C(J ; \mathbb{R})$ defined by the relation

$$
(\mathcal{F} y)(t)=f(t)+(A y)(t) \int_{0}^{T} u(t, s, y(s), y(\alpha s)) d s, \quad t \in J .
$$

Let $y$ be a possible solution of the problem (1.1). For given $n \in \mathbb{N}$ and $t \leq n$, in view of $\left(a_{1}\right),\left(a_{2}\right)$, and $\left(a_{5}\right)$, we have

$$
\begin{aligned}
|y(t)| & \leq|f(t)|+|(A y)(t)| \int_{0}^{T}|u(t, s, y(s), y(\alpha s))| d s \\
& \leq|f(t)|+(a+b|y(t)|) \int_{0}^{T} p(s) \psi(|y(s)|,|y(\alpha s)|) d s \\
& \leq\|f\|_{n}+T\left(a+b\|y\|_{n}\right) \psi\left(\|y\|_{n},\|y\|_{n}\right) p^{*}
\end{aligned}
$$

and thus

$$
\frac{\|y\|_{n}}{\|f\|_{n}+T\left(a+b\|y\|_{n}\right) \psi\left(\|y\|_{n},\|y\|_{n}\right) p^{*}} \leq 1 .
$$

From (3.1) it follows that $\|y\|_{n} \neq M_{n}$ for each $n \in \mathbb{N}$. Now, set

$$
\Omega=\left\{y \in(J ; \mathbb{R}):\|y\|_{n}<M_{n} \text { for every } n \in \mathbb{N}\right\} .
$$

Clearly, $\Omega$ is an open subset of $C(J ; \mathbb{R})$. We shall show that $\mathcal{F}: \bar{\Omega} \rightarrow C(J ; \mathbb{R})$ is a contraction operator. Indeed, consider $y, \bar{y} \in C(J ; \mathbb{R})$, for each $t \in[0, n]$ and $n \in \mathbb{N}$, from $\left(a_{2}\right)-\left(a_{5}\right)$ we get

$$
\begin{aligned}
& |(\mathcal{F} y)(t)-(\mathcal{F} \bar{y})(t)| \\
& \leq\left|(A y)(t) \int_{0}^{T} u(t, s, y(s), y(\alpha s)) d s-(A \bar{y})(t) \int_{0}^{T} u(t, s, \bar{y}(s), \bar{y}(\alpha s)) d s\right| \\
& \leq\left|(A y)(t) \int_{0}^{T} u(t, s, y(s), y(\alpha s)) d s-(A y)(t) \int_{0}^{T} u(t, s, \bar{y}(s), \bar{y}(\alpha s)) d s\right| \\
& \quad+\left|(A y)(t) \int_{0}^{T} u(t, s, \bar{y}(s), \bar{y}(\alpha s)) d s-(A \bar{y})(t) \int_{0}^{T} u(t, s, \bar{y}(s), \bar{y}(\alpha s)) d s\right|
\end{aligned}
$$




$$
\begin{aligned}
\leq & |(A y)(t)| \int_{0}^{T}|u(t, s, y(s), y(\alpha s))-u(t, s, \bar{y}(s), \bar{y}(\alpha s))| d s \\
& +|(A y)(t)-(A \bar{y})(t)| \int_{0}^{T}|u(t, s, \bar{y}(s), \bar{y}(\alpha s))| d s \\
\leq & (a+b|y(t)|) L_{n}^{*} \int_{0}^{T}(|y(s)-\bar{y}(s)|+|y(\alpha s)-\bar{y}(\alpha s)|) d s \\
& +L_{n}|y(t)-\bar{y}(t)| \int_{0}^{T} p(s) \psi(|\bar{y}(s)|,|\bar{y}(\alpha s)|) d s \\
\leq & {\left[2\left(a+b M_{n}\right) L_{n}^{*} T+T L_{n} \psi\left(M_{n}, M_{n}\right) p^{*}\right]\|y-\bar{y}\|_{n} . }
\end{aligned}
$$

Therefore,

$$
\|\mathcal{F} y-\mathscr{F} \bar{y}\|_{n} \leq\left[2\left(a+b M_{n}\right) L_{n}^{*} T+T L_{n} \psi\left(M_{n}, M_{n}\right) p^{*}\right]\|y-\bar{y}\|_{n} .
$$

So by (3.2) the operator $\mathscr{F}$ is a contraction for all $n \in \mathbb{N}$. From the choice of $\Omega$ there is no $y \in \partial \Omega$ such that $y=\lambda \mathscr{F}(y)$ for some $\lambda \in(0,1)$. Then the statement (C2) in Theorem 2.1 does not hold. A consequence of the Leray-Schauder type nonlinear alternative from [12] yields that $(\mathrm{C} 1)$ holds and thus we deduce that the operator $\mathcal{F}$ has a unique fixed-point $y$ in $\bar{\Omega}$, which is a solution to Equation (1.1). This completes the proof.

Theorem 3.2. Let the following assumptions be satisfied:

$\left(\hat{a}_{1}\right) f: J \rightarrow \mathbb{R}$ is a continuous function.

$\left(\hat{a}_{2}\right) g: J \times \mathbb{R} \rightarrow \mathbb{R}$ is continuous and, for each $n \in \mathbb{N}$, there exists $L_{n}>0$ such that

$$
|g(t, x)-g(t, \bar{x})| \leq L_{n}|x-\bar{x}| \quad \text { for all } x, \bar{x} \in \mathbb{R} \text { and } t \in[0, n] .
$$

$\left(\hat{a}_{3}\right) u: J \times J_{T} \times \mathbb{R}^{2} \rightarrow \mathbb{R}$ is a continuous function and, for each $n \in \mathbb{N}$, there exists a constant $L_{n}^{*}>0$ such that

$$
|u(t, s, x, y)-u(t, s, \bar{x}, \bar{y})| \leq L_{n}^{*}(|x-\bar{x}|+|y-\bar{y}|)
$$

holds for all $(t, s) \in[0, n] \times J_{T}$ and $x, y, \bar{x}, \bar{y} \in \mathbb{R}$.

$\left(\hat{a}_{4}\right)$ There exists a continuous nondecreasing function $\psi: J^{2} \rightarrow(0, \infty)$ and $p \in$ $C\left(J ; \mathbb{R}_{+}\right)$such that

$$
|u(t, s, x, y)| \leq p(s) \psi(|x|,|y|)
$$

for each $(t, s) \in J \times J_{T}$ and $x, y \in \mathbb{R}$ and, moreover, there exists constants $M_{n} \in J, n \in \mathbb{N}$, such that

$$
\frac{M_{n}}{\|f\|_{n}+T\left(L_{n} M_{n}+m_{n}\right) \psi\left(M_{n}, M_{n}\right) p^{*}}>1
$$

holds for all $n \in \mathbb{N}$, where $p^{*}=\sup \left\{p(s): s \in J_{T}\right\}$ and $m_{n}=\sup \{g(t, 0)$ : $t \in[0, n]\}$. 
If, in addition, the inequality

$$
2\left(L_{n} M_{n}+m_{n}\right) T L_{n}^{*}+T L_{n} \psi\left(M_{n}, M_{n}\right) p^{*}<1
$$

holds, then Equation (1.2) has a unique solution.

Proof. The proof is similar to those of Theorem 3.1.

Example 1. Consider the quadratic integral equation of the Urysohn type

$$
x(t)=1+\frac{|x(t)|}{1+|x(t)|} \int_{0}^{T} \frac{t s}{t^{3}+1}\left(x(s)+x\left(\frac{s}{2}\right)\right) d s, t \in J:=[0,+\infty) .
$$

Set $f(t)=1$ for each $t \in J, \psi(x, y)=x+y$ for all $x, y \geq 0$,

$$
(A x)(t)=\frac{|x(t)|}{1+|x(t)|}, \quad t \in J \text { and } x \in C(J ; \mathbb{R}),
$$

and

$$
u(t, s, x, y)=\frac{t s}{t^{3}+1}(x+y)
$$

for all $(t, s) \in J \times J_{T}$ and $x, y \in \mathbb{R}$. It is clear that (3.4) is a particular case of equation (1.1). Let us show that conditions $\left(a_{1}\right)-\left(a_{5}\right)$ hold.

For each $n \in \mathbb{N}, t \in[0, n]$, and $x, \bar{x} \in C\left(J ; \mathbb{R}_{+}\right)$, we have

$$
\begin{aligned}
|(A x)(t)-(A \bar{x})(t)| & =\left|\frac{|x(t)|}{1+|x(t)|}-\frac{|\bar{x}(t)|}{1+|\bar{x}(t)|}\right|=\left|\frac{|x(t)|-|\bar{x}(t)|}{(1+|x(t)|)(1+|\bar{x}(t)|) \mid}\right| \\
& \leq \frac{|x(t)-\bar{x}(t)|}{(1+|x(t)|)(1+|\bar{x}(t)|)} \leq|x(t)-\bar{x}(t)| .
\end{aligned}
$$

Hence $\left(a_{2}\right)$ is satisfied with $L_{n}=1$.

For each $t \in J$ and $x \in C(J ; \mathbb{R})$, we have

$$
|(A x)(t)|=\left|\frac{|x(t)|}{1+|x(t)|}\right| \leq|x(t)| .
$$

Hence $\left(a_{3}\right)$ holds with $a=0$ and $b=1$.

For each $n \in \mathbb{N},(t, s) \in[0, n] \times J_{T}$, and $x, y, \bar{x}, \bar{y} \in \mathbb{R}$, we have

$$
\begin{aligned}
|u(t, s, x, \bar{x})-u(t, s, y, \bar{y})| & =\left|\frac{t s}{t^{3}+1}[(x+\bar{x})-(y+\bar{y})]\right| \\
& \leq\left|\frac{t s}{t^{3}+1}[(x-y)+(\bar{x}-\bar{y})]\right| \\
& \leq \frac{n T}{n^{3}+1}[|x-y|+|\bar{x}-\bar{y}|] \\
& \leq T[|x-y|+|\bar{x}-\bar{y}|] .
\end{aligned}
$$

Hence $\left(a_{4}\right)$ is satisfied with $L_{n}^{*}=T$. 
For each $n \in \mathbb{N},(t, s) \in[0, n] \times J_{T}$, and $x, y \in \mathbb{R}$, we have

$$
\begin{aligned}
|u(t, s, x, y)| & =\left|\frac{t s}{t^{3}+1}(x+y)\right| \\
& \leq s(|x|+|y|)=s \psi(|x|,|y|) .
\end{aligned}
$$

To conclude that $\left(a_{5}\right)$ holds we shall show that (3.1) is satisfied. Indeed

$$
\begin{aligned}
\frac{M_{n}}{\|f\|_{n}+T\left(a+b M_{n}\right) \psi\left(M_{n}, M_{n}\right) p^{*}}>1 & \Longleftrightarrow \frac{M_{n}}{1+2 T^{2} M_{n}^{2}}>1 \\
& \Longleftrightarrow 2 T^{2} M_{n}^{2}-M_{n}+1<0 .
\end{aligned}
$$

Notice that the last inequality holds for $T$ such that $1-8 T^{2}>0$, i.e.,

$$
T<\frac{1}{2 \sqrt{2}} \text {. }
$$

Hence for $T>0$ satisfying (3.5), there exists $M_{n}>0$ satisfying (3.1).

Finally let us show that (3.2) is satisfied.

$$
\begin{aligned}
2\left(a+b M_{n}\right) L_{n}^{*} T+T L_{n} \psi\left(M_{n}, M_{n}\right) p^{*}-1 & =2 M_{n} T^{2}+2 T^{2} M_{n}-1 \\
& =4 M_{n} T^{2}-1 .
\end{aligned}
$$

Hence (3.2) is satisfied for $T$ or $M_{n}$ satisfying $4 M_{n} T^{2}-1<0$, i. e., for

$$
0<T<\frac{1}{2 \sqrt{M_{n}}}
$$

or $0<M_{n}<\frac{1}{4} T^{-2}$. Consequently, if $T$ satisfies the inequalities

$$
0<T<\min \left\{\frac{1}{2 \sqrt{M_{n}}}, \frac{1}{2 \sqrt{2}}\right\},
$$

then it follows from Theorem 3.1 that equation (3.4) has a unique solution.

\section{ACKNOWLEDGEMENT}

This work was completed when the authors were visiting the ICTP in Trieste as Regular Associates. It is a pleasure for them to express gratitude for its financial support and the warm hospitality.

\section{REFERENCES}

[1] R. P. Agarwal and D. O'Regan, Infinite interval problems for differential, difference and integral equations. Dordrecht: Kluwer Academic Publishers, 2001.

[2] R. P. Agarwal, D. O'Regan, and P. J. Y. Wong, Positive solutions of differential, difference and integral equations. $\quad$ Dordrecht: Kluwer Academic Publishers, 1999.

[3] J. Banaś, J. Caballero, J. Rocha, and K. Sadarangani, "Monotonic solutions of a class of quadratic integral equations of Volterra type," Comput. Math. Appl., vol. 49, no. 5-6, pp. 943-952, 2005. 
[4] M. Benchohra and M. A. Darwish, "On monotonic solutions of a quadratic integral equation of Hammerstein type," Intern. J. Appl. Math. Sci., to appear.

[5] J. Caballero, B. López, and K. Sadarangani, "Existence of nondecreasing and continuous solutions of an integral equation with linear modification of the argument," Acta Math. Sin. (Engl. Ser.), vol. 23, no. 9, pp. 1719-1728, 2007.

[6] J. Carr and J. Dyson, "The functional differential equation $y^{\prime}(x)=a y(\lambda x)+b y(x)$," Proc. Roy. Soc. Edinburgh Sect. A, vol. 74, pp. 165-174 (1976), 1974/75.

[7] S. Chandrasekhar, Radiative transfer. New York: Dover Publications Inc., 1960.

[8] C. Corduneanu, Integral equations and applications. Cambridge: Cambridge University Press, 1991.

[9] M. A. Darwish, "On quadratic integral equation of fractional orders," J. Math. Anal. Appl., vol. 311, no. 1, pp. 112-119, 2005.

[10] K. Deimling, Nonlinear functional analysis. Berlin: Springer-Verlag, 1985.

[11] G. M. Dunkel, "Function differential equations: examples and problems," in Seminar on Differential Equations and Dynamical Systems, II (Univ. Maryland, College Park, Md., 1969). Berlin: Springer, 1970, pp. 49-63. Lecture Notes in Math., Vol. 144.

[12] M. Frigon and A. Granas, "Résultats de type Leray-Schauder pour des contractions sur des espaces de Fréchet," Ann. Sci. Math. Québec, vol. 22, no. 2, pp. 161-168, 1998, dedicated to the memory of Gilles Fournier (Sherbrooke, PQ, 1997).

[13] M. Heard, "A family of solutions of the IVP for the equation $x^{\prime}(t)=a x(\lambda t), \lambda>1$," Aequationes Math., vol. 9, pp. 273-280, 1973.

[14] S. Hu, M. Khavanin, and W. Zhuang, "Integral equations arising in the kinetic theory of gases," Appl. Anal., vol. 34, no. 3-4, pp. 261-266, 1989.

[15] C. T. Kelley, "Approximation of solutions of some quadratic integral equations in transport theory," J. Integral Equations, vol. 4, no. 3, pp. 221-237, 1982.

[16] M. R. S. Kulenović, "Oscillation of the Euler differential equation with delay," Czechoslovak Math. J., vol. 45(120), no. 1, pp. 1-6, 1995.

[17] R. W. Leggett, "A new approach to the $H$-equation of Chandrasekhar," SIAM J. Math. Anal., vol. 7, no. 4, pp. 542-550, 1976.

[18] V. Mureşan, "On a class of Volterra integral equations with deviating argument," Studia Univ. Babeş-Bolyai Math., vol. 44, no. 1, pp. 47-54, 1999.

[19] V. Mureşan, "Volterra integral equations with iterations of linear modification of the argument," Novi Sad J. Math., vol. 33, no. 2, pp. 1-10, 2003.

[20] D. O'Regan and M. Meehan, Existence theory for nonlinear integral and integrodifferential equations, ser. Mathematics and its Applications. Dordrecht: Kluwer Academic Publishers, 1998, vol. 445 .

[21] C. A. Stuart, "Existence theorems for a class of non-linear integral equations," Math. Z., vol. 137, pp. 49-66, 1974.

\section{Authors' addresses}

\section{Mouffak Benchohra}

Laboratoire de Mathématiques, Université de Sidi Bel-Abbès, BP 89, 22000 Sidi Bel-Abbès, Algérie

E-mail address: benchohra@yahoo.com

\section{Mohamed Abdalla Darwish}

Department of Mathematics, Faculty of Science, Alexandria University, Damanhour Branch, 22511

Damanhour, Egypt

E-mail address: darwishma@yahoo.com, mdarwish@ictp.trieste.it 